\title{
Challenges in the management of corneal ulcer
}

\author{
Singh SK \\ Associate Professor and Medical Director, \\ Biratnagar Eye Hospital, Nepal
}

Corneal scar due to various corneal diseases is a major cause of blindness. Its epidemiology encompasses a wide variety of infectious and inflammatory eye diseases (Whitchr et al, 2001). The prevalence of corneal blindness varies from country to country and even from one population to another, depending on many factors, such as the availability and general standards of eye care (Smith et a, 1991). In a study from South India, prevalence of corneal blindness was $0.66 \%$ in at least one eye, which included $0.10 \%$ prevalence of corneal blindness in both eyes. Corneal ulceration and trauma were the two major causes of corneal blindness in APEDS (R Dandona et al., 2003). In the Nepal blindness survey, corneal trauma and ulceration were found to be the second leading cause of unilateral visual loss after cataract, accounting for $7.9 \%$ of all blind eyes (Brilliant LB et al, 1985). The prevalence of blindness worldwide secondary to microbial keratitis is currently unknown. Recent evidence suggests, however, that corneal ulceration is a much more common event than previously recognized and is a major cause of corneal scarring and visual loss in developing countries. The incidence of corneal ulceration in Nepal is one of the highest reported in the world. The Bhaktapur Eye Study revealed it to be 799 per 100,000 population per year (Upadhyay et al, 2001), which is seven times higher than in South India (Gonzales CA et al, 1996), and seventy times greater than reported in the USA (Erie JC et al, 1993).

Treatment of corneal ulcer is easier said than done for various reasons. Usually, the patients affected are poor people in the developing world (Whitcher et al, 2002) who are at a higher risk for microbial keratitis because of their occupational exposure and their inability to access the existing health care system. In a study done in the Eastern Region of Nepal, patient's accessibility to eye care services for the treatment of corneal ulcer was the main barrier to the uptake of eye services followed by cost, social belief and ignorance about the disease (Singh S K, 2004-2005). Other barriers included a distant location of eye care services, no/ poor transportation services, a busy agricultural season, and late referrals from health centers and private practitioners.

The location of tertiary eye hospitals and eye departments in the Terai part of the Eastern Region of Nepal makes eyecare services more accessible to the people of this region. A corneal ulcer patient in the hilly and mountainous regions has to walk several hours or even days to catch a bus to reach a tertiary eye care centre. At the same time, as the transportation cost is more expensive in the hilly areas, the patient has to pay more for his travel compared to the people from the Terai region of the country. People living in the urban areas of Nepal and from the bordering area of India have better accessibility to tertiary eye hospitals in Nepal due to the relatively better conditions of roads, an a available rail network (in India), and comparatively cheaper fares for transportation services.

The corneal ulcer patients face both direct and indirect costs while getting treatment. Indirect costs like the loss of daily wages of both the patients and their attendants and direct costs like the cost of medicine and the expenditure incurred during a long stay in hospital increases the overall cost of treatment. In a study done in South India (Ramalingam et al, 1998-1999), belief in traditional eye medicine and cost were the major barriers preventing patients from seeking early treatment for corneal ulcer at eye hospitals. Due to different barriers faced by them, many patients are losing the initial critical period for the management of corneal abrasion/ ulcer and are accessing some harmful forms of treatment.

The main barrier from the service provider group for corneal ulcer management is lack of diagnostic facilities. In the Eastern Region of Nepal, with a population of over 5 million, the facility for culture for corneal ulcer patients exists only at the eye department of the B P Koirala Institute of Health Sciences in Koshi Zone. None of the other tertiary eye hospital have laboratory facilities with the availability of culture and sensitivity tests for the management of corneal ulcer. The existing laboratory facilities in different government health institutions (health posts, health centers, district hospitals, and zonal hospitals) are not utilized for the 
management of corneal ulcers because of the lack of ophthalmic manpower. Additionally, the lack of training for the existing health manpower in government health care centers is a significant factor resulting in the improper management of corneal ulcers. There is also a lack of co-ordination between existing government health structures and eye-care infrastructures run by non-governmental organizations.

Cataract oriented eye programs, the large number of cataract patients, and a relatively low number of corneal ulcer patients in the existing tertiary eye hospitals could be the factors responsible for the lack of development of laboratory facilities in these hospitals. An ample cataract population, a greater interest of ophthalmic surgeons in strengthening their cataract surgery skills could be the reasons for ophthalmologists being more interested in doing cataract surgeries and having both less interest and less time for the management of corneal ulcers in some hospitals. The same reasons may apply to hospital administration people being less interested in the development of facilities for management of corneal ulcers. In many developing countries, because of the emphasis on the cataract backlog, a programme dealing with other causes of blindness has been neglected (Dandona L et al, 1988). Probably the same is true in Nepal as well.

It is advisable that a combined approach be made with efforts of all the stakeholders of eye-care in the region to minimize the stubborn prevalence of corneal blindness in general and of corneal ulcer in particular.

\section{References}

Brilliant LB, Pokharel RP, Grosset NC, Lepkowski J (1985). Epidemiology of blindness in Nepal. Bull WHO;63:375-386.

Dandona L et al (1988). Is current eye-care policy focused almost exclusively on cataract adequate to deal with blindness in India? Lancet; 351: 1312-1316.

Erie JC et al (1993). Incidence of ulcerative keratitis in a defined population from 1950 through 1988. Archieves of Ophthalmology; 111: 1665-1671.

Gonzales CA et al (1996). Incidence of corneal ulceration in Madurai District, South India. Ophthalmic Epidemiology; 3: 159-166.

Whitchr J P, Srinivasan M, Upadhyay MP (2001). Corneal Blindness: a global perspective. Bulletin of the World Health Organization; 79 (3).

Upadhyay MP, Karmacharya PC and Koirala S et al (2001). The Bhaktapur Eye Study: ocular trauma and antibiotic prophylaxis for the prevention of corneal ulceration in Nepal. British Journal of Ophthalmology; 85:388-392.
Ramalingam MDK . A qualitative analysis of barriers to early up-take of treatment of corneal ulcers. MSc Dissertation (submitted in partial fulfilment for the degree of MSc in Community Eye Health, University College London, 1998-1999.

Dandona R , Dandona L (2003). Corneal blindness in a southern Indian population: need for health promotion strategies. British Journal of Ophthalmology; 87:133-141.

Singh S K. Existing practice pattern and barriers of corneal ulcers management in the eastern region of Nepal, M.Sc. Community Eye Health Dissertation, London School of Hygiene and Tropical Medicine, 2004-2005.

Smith GTH, Taylor HR (1991). Epidemiology of corneal blindness in developing countries. Refractive and Corneal Surgery; 7: 436-439.

Whitcher, JP, Srinivasan M, Upadhyay M P (2002). Prevention of corneal ulceration in the developing world. International Ophthalmology Clinics: 42(1):7177. 